\title{
PAPER \\ Optimum Wavelength Filter Spectrum Response in DWDM Systems for Ultimate Spectral Efficiency
}

Shuichi SUZUKI ${ }^{\dagger *}$ and Yasuo KOKUBUN ${ }^{\dagger a)}$, Members

SUMMARY A method of evaluating the wavelength filter spectrum response is introduced. The increase of the crosstalk level due to the filtering and the relation between the total crosstalk and the spectral efficiency are derived in detail using the Gaussian filter. Since this method can be applied to various kinds of filter spectrum responses, the ultimate spectral efficiencies of filters are compared. In this comparison, the problem of the box-like filter, which has been considered to be desirable, is revealed, and this is improved by cascading the filter spectrum. The requirement on the rejection floor that inheres in the filter is also made clear.

key words: WDM, wavelength multi/demultiplexer, spectral efficiency, crosstalk, cascaded filter, crosstalk floor

\section{Introduction}

In the DWDM system, further increase of transmission capacity of the optical fiber transmission is demanded by the explosive increase of the capacity demand due to the rapid and widespread diffusion of the Internet. One of the methods to increase the transmission capacity in the DWDM systems is the increase of the spectral efficiency, that is, the bit rate per unit wavelength range. The number of channels is increased by narrowing the channel spacing, and so the transmission capacity in a given wavelength range increases. This is equivalent to the increase of the spectral efficiency. Thus, the total transmission capacity is increased by increasing the spectral efficiency if the bit rate per channel is kept constant.

In the ultra DWDM transmission over $10 \mathrm{Tbit} / \mathrm{sec}$ [1] [3], the ultimate spectral efficiency is required to maximize the transmission capacity in a given wavelength range. Although the limit of spectral efficiency in the DWDM system has been reported by Ho and Kahn [4], the limit is calculated under the condition of zero error according to the Shannon's theory [5], and there is no report on the effect of crosstalk between adjacent channels in the DWDM transmission systems. On the other hand, the theoretical limit of the spectral efficiency of unity has been reported by Nyquist [6]. However, this corresponds to the case of signal spectrum with perfect rectangular (box-like) shape. Although this can be realized by an ultra-short (very wide-band) pulse signal and box-like wavelength filter, the pulse shape is a so-called sink function. In this case, tolerance against the timing jitter is

\footnotetext{
Manuscript received July 5, 2004.

Manuscript revised January 11, 2005.

${ }^{\dagger}$ The authors are with Yokohama National University, Yokohama-shi, 240-8501 Japan.

*Presently, with Ricoh Corp.

a)E-mail: kokubun@ynu.ac.jp

DOI: 10.1093/ietcom/e88-b.9.3649
}

quite small, because the intersymbol interference is eliminated by careful adjustment of the timing of the pulse interval to the null point of the sink function. In addition, the perfect box-like filter means that the out-of-band rejection is infinity. However, this can not be realized because the infinite rejection violates the causality. Therefore, we need to take into account the crosstalk from adjacent wavelength channels in the actual WDM system.

The spectral efficiency is limited by the crosstalk due to the partition separation of the frequency domain, and this crosstalk level is dependent on the spectrum response of the wavelength filter (multi/demultiplexer) used in the DWDM system. Therefore we have clarified the optimum allocation of optical signals and filter responses both in the time and frequency domains, taking into account the coherent crosstalk caused by the interference of signals between adjacent wavelength channels [7].

In our previous analysis [7], an intersymbol interference in the time domain and the interchannel crosstalk in the frequency domain were considered as the crosstalk, and the optimum crosstalk condition was defined so that these levels were equal, because these crosstalks took trade-off relation to the input pulse width. Defining this optimum crosstalk condition, the spectral efficiency and the total crosstalk level could be related using the parameters concerning only the filter response. Using this analysis method, the ultimate spectral efficiency for a given crosstalk level was obtained, and the evaluation of the filter spectrum response was made possible from the viewpoint of the ultimate spectral efficiency. Although we demonstrated the optimum design of filter bandwidth and input pulse width to obtain the maximum spectral efficiency for ideal Gaussian filter response [6], we did not consider other filter spectrum responses to increase the spectral efficiency or the impact of a rejection floor on the spectral efficiency, which is inherent in the wavelength filter.

In this study, we analyzed the relation between the spectral efficiency and the crosstalk level for the box-like filter spectrum response, which is generally considered to be desirable to obtain a high spectral efficiency. Additionally, we clarified the requirement on the rejection floor level from the viewpoint of the degradation of the spectral efficiency.

In this analysis, the following conditions were assumed; (a) Gaussian input single pulse, (b) the dispersion of the transmission line is compensated for at the input port of the filter, (c) the dispersion of the filter is compensated for at the output port, (d) RZ format, IM/DD, (e) there is 
no polarization multiplexing, and (f) the peak powers of all channels are equal. Although in the actual transmission system the dispersion and some nonlinear effects of the fiber affect the transmission characteristics, there remains a limit of transmission capacity in the DWDM system, even if the transmitter and the receiver are directly connected without the fiber transmission line. Thus we intend to clarify the ultimate spectral efficiency limited by the partition of frequency channels in the DWDM system.

\section{Considered Crosstalks}

In this analysis, two kinds of crosstalks, the intersymbol interference and the interchannel crosstalk were considered as shown in Fig. 1. Figure 1(a) shows the crosstalk in the frequency domain, i.e. the interchannel crosstalk. Let us suppose that the signal in channel \#1 leaks to channel \#2. Then the product of the signal spectrum (dashed curve) and the filter response curve (solid curve) corresponds to the spectrum of crosstalk (dash-dotted curve). The crosstalk level is measured by the difference between the peak of the crosstalk spectrum and the peak level of channel \#2. On the other hand, Fig. 1(b) shows the crosstalk in the time domain, i.e. the intersymbol interference. This occurs in the same wavelength channel, and the crosstalk is defined by the power level of the preceding pulse at the center of timing of the signal pulse. The interchannel crosstalk $C T^{(v)}$ is the signal leakage from adjacent ports and depends on the input pulse width $\delta t_{s}$ (spectrum width $\delta v_{s}$ ), the spectrum width of filter $\delta v_{f}$ (FWHM of impulse response $\delta t_{f}$ ), and the channel spacing $\Delta v$. The intersymbol interference $C T^{(T)}$ is the time domain crosstalk in a fixed wavelength channel and depends on $\delta t_{s}\left(\delta v_{s}\right), \delta v_{f}\left(\delta t_{f}\right)$, and the pulse interval $\Delta T$ (bit rate $1 / \Delta T)$. First, the intersymbol interference $C T^{(T)}$ originating from the preceding pulse is calculated from $f_{\text {out }}(\Delta T)$. Next, considering the interchannel crosstalk $C T^{(v)}$, the output pulse amplitude from the filter $f_{\text {out }}(t)$ includes the channel signal $f_{\text {out }}^{(s)}(t)$ and the leaked crosstalk from adjacent ports $f_{\text {out }}^{( \pm \delta v)}(t)$. Thus, the total output power includes the leaked power crosstalk $C T^{(L P)}$ from adjacent channels and the coherent crosstalk $C T^{(C C)}$ resulting from the interference between $f_{\text {out }}^{(s)}(t)$ and $f_{\text {out }}^{( \pm \delta v)}(t)$.

We analyzed a Gaussian filter response, because we could obtain a strict analytical expression for the output

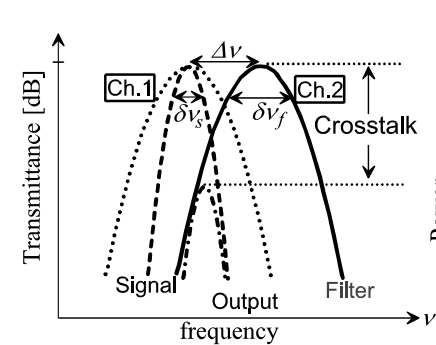

(a) interchannel crosstalk

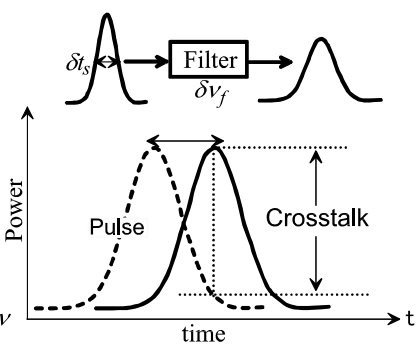

(b) intersymbol interference
Fig. 1 Definition of crosstalks in frequency and time domains. pulse using the Fourier transform. In addition, the filter response of conventional AWGs can be approximated well by the Gaussian function, although some modifications are added to obtain the flat-top spectrum shape for practical purpose. Let us define an input pulse by

$$
f_{\text {in }}(t)=\exp \left(-a t^{2}\right) \cdot \exp \left(j \omega_{0} t\right),
$$

where $\omega_{0}$ is the angular frequency of the optical carrier. Using the Fourier transform, the input spectrum is given by

$$
F_{\text {in }}(\omega)=\sqrt{\frac{\pi}{a}} \exp \left[-\frac{\left(\omega-\omega_{0}\right)^{2}}{4 a}\right] .
$$

Here, the FWHMs of the pulse and the spectrum $\delta t_{s}$ and $\delta v_{s}$ are related in terms of the coefficient $a$ by

$$
\left(\frac{\delta t_{s}}{2}\right)^{2}=\frac{\ln \sqrt{2}}{a}=\frac{\ln 2}{2 a}
$$

and

$$
\left(\pi \cdot \delta v_{s}\right)^{2}=\left(\frac{\delta \omega_{s}}{2}\right)^{2}=\ln \sqrt{2} \cdot 4 a=2 a \cdot \ln 2,
$$

respectively.

Next, let us define a filter spectrum response by

$$
H(\omega)=\exp \left[-\frac{\left(\omega-\omega_{0}\right)^{2}}{4 b}\right]
$$

where $\omega_{0}$ is the center angular frequency and $2 \sqrt{b}$ is the FWHM of the spectrum response.

The output spectrum $F_{\text {out }}^{(s)}(\omega)$ and the output pulse $f_{\text {out }}^{(s)}(t)$ are calculated as follows:

$$
\begin{aligned}
F_{\text {out }}^{(s)}(\omega) & =F_{\text {in }}(\omega) \cdot H(\omega) \\
& =\sqrt{\frac{\pi}{a}} \exp \left[-\left(\frac{1}{a}+\frac{1}{b}\right) \frac{\left(\omega-\omega_{0}\right)^{2}}{4}\right]
\end{aligned}
$$

and

$$
f_{\text {out }}^{(s)}(t)=\frac{1}{\sqrt{1+\frac{a}{b}}} \exp \left[-\frac{1}{\frac{1}{a}+\frac{1}{b}} t^{2}\right] \cdot \exp \left(j \omega_{0} t\right),
$$

respectively.

Using these definitions, the crosstalk in the time domain $C T^{(T)}$ is given by

$$
\begin{aligned}
C T^{(T)} & =\left|\exp \left[-\frac{1}{\frac{1}{a}+\frac{1}{b}} \Delta T^{2}\right]\right|^{2} \\
& =\left|\exp \left[-\frac{4 \ln \sqrt{2}}{\left(\frac{\delta t_{s}}{\Delta T}\right)^{2}+\left(\frac{\delta t_{f}}{\Delta T}\right)^{2}}\right]\right|^{2} .
\end{aligned}
$$

Also, the crosstalk in the frequency domain is calculated. In the frequency domain, we need to consider two kinds of crosstalks: the crosstalk by the leakage power from one adjacent channel $C T^{(L P)}$, which is given by

$$
C T^{(L P)}=\left|\exp \left[-\frac{\Delta \Omega^{2}}{4 a+4 b}\right]\right|^{2}
$$


Table 1 Classification of crosstalk.

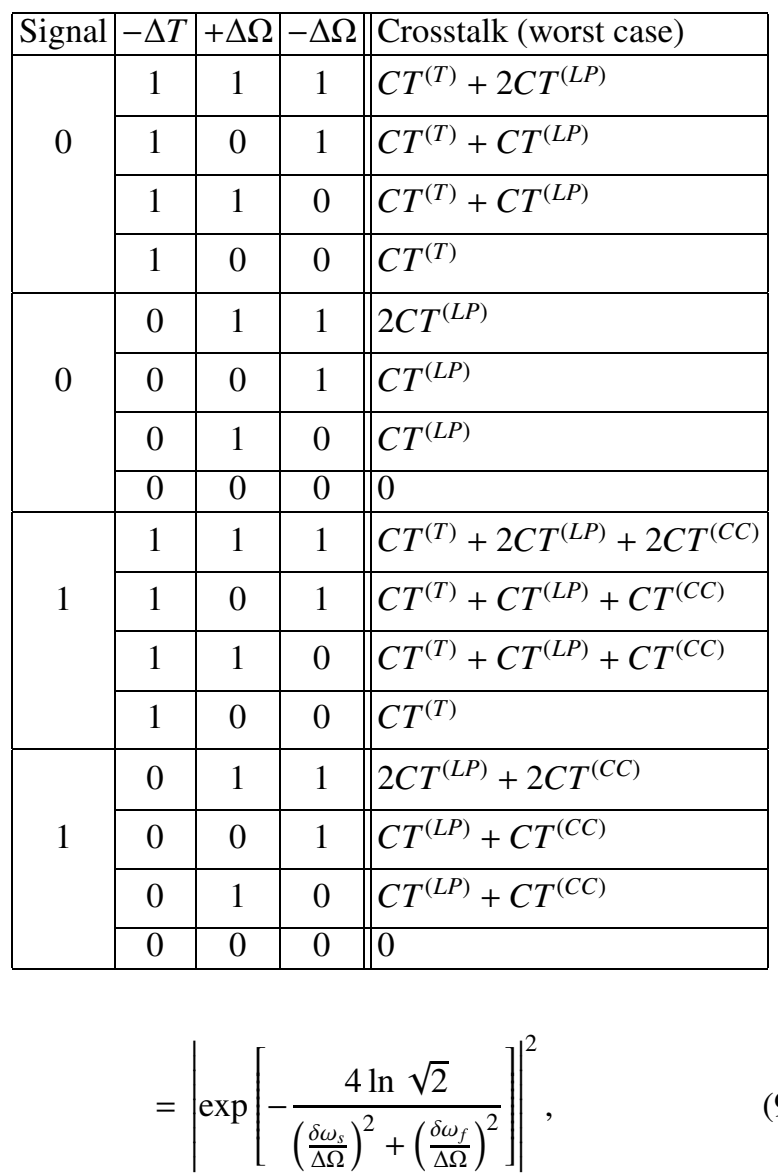

and the coherent crosstalk by the interference between the signal channel and adjacent channels $C T^{(C C)}$, which is given by

$$
C T^{(C C)}=4 \exp \left[-\frac{4 \ln \sqrt{2}}{\left(\frac{\delta \omega_{s}}{\Delta \Omega}\right)^{2}+\left(\frac{\delta \omega_{f}}{\Delta \Omega}\right)^{2}}\right]
$$

The derivation of Eqs. (8)-(10) is given in Appendix.

The intersymbol interference and the interchannel crosstalk can be categorized as shown in Table 1 .

\section{Optimum Crosstalk Condition and Ultimate Spec- tral Efficiency}

Figure 2 shows the relation between the crosstalks and the input pulse width. The intersymbol interference and interchannel crosstalk have a trade-off relation to the input pulse width. Here, we assume the optimum crosstalk condition is given by $C T^{(v)}=C T^{(T)}$ for the worst case of $C T^{(v)}$. Then we can obtain the relation between the total crosstalk level and the spectral efficiency.

\subsection{Crosstalk by Leakage Power}

First, we assume the leakage power from adjacent $\pm \Delta \Omega$ channels as the interchannel crosstalk. In this case, the optimum crosstalk condition is given by $C T^{(T)}=2 C T^{(L P)}$.

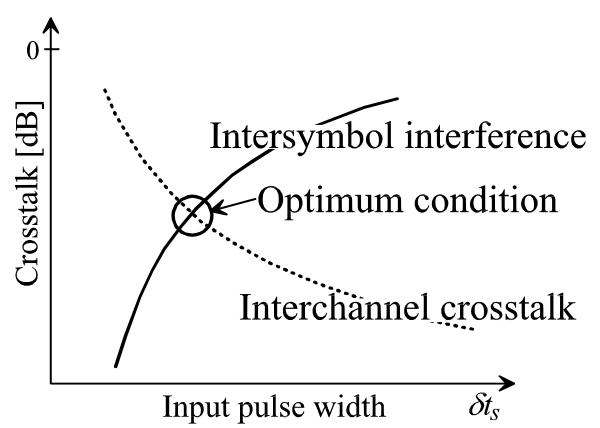

Fig. 2 Definition of optimum crosstalk condition.

Therefore, the following equation is derived by substituting Eqs. (8) and (9) to the above equation:

$$
\left|\exp \left[-\frac{4 \ln \sqrt{2}}{\left(\frac{\delta t_{s}}{\Delta T}\right)^{2}+\left(\frac{\delta t_{f}}{\Delta T}\right)^{2}}\right]\right|^{2}=2\left|\exp \left[-\frac{4 \ln \sqrt{2}}{\left(\frac{\delta \omega_{s}}{\Delta \Omega}\right)^{2}+\left(\frac{\delta \omega_{f}}{\Delta \Omega}\right)^{2}}\right]\right|^{2} .
$$

By solving this equation and representing $\frac{\delta \omega_{s}}{\Delta \Omega}$ by $\frac{\delta t_{s}}{\Delta T}$ using Eqs. (3) and (4), we obtain the optimum condition as

$$
\left(\frac{\delta t_{s}}{\Delta T}\right)^{2}=\frac{\left(\frac{\delta \omega_{f}}{\Delta \Omega}\right)^{2}\left[4+\left(\frac{\delta t_{f}}{\Delta T}\right)^{2}\right]}{4-\left(\frac{\delta \omega_{f}}{\Delta \Omega}\right)^{2}}
$$

Since the total crosstalk value is given by $2\left|\exp \left[-\frac{4 \ln \sqrt{2}}{\left(\frac{\delta t_{s}}{\Delta T}\right)^{2}+\left(\frac{\delta t_{f}}{\Delta T}\right)^{2}}\right]\right|^{2}$, substituting Eq. (12) into this formula, we can express the total crosstalk in terms of $\frac{\delta \omega_{f}}{\Delta \Omega}$ and $\frac{\delta t_{f}}{\Delta T}$, i.e. only the parameters of the filter spectrum. After some calculation, the relation between $\frac{\delta \omega_{f}}{\Delta \Omega}$ and $\frac{\delta t_{f}}{\Delta T}$ is obtained by

$$
\begin{aligned}
\left(\frac{\delta t_{f}}{\Delta T}\right)^{2}= & \frac{40 \log 2}{10 \log 2-C T^{(d B)}} \\
& -\left(\frac{\delta \omega_{f}}{\Delta \Omega}\right)^{2}\left(\frac{10 \log 2}{10 \log 2-C T^{(d B)}}+1\right),
\end{aligned}
$$

where $C T^{(d B)}$ is the total crosstalk level in $\mathrm{dB}$. Therefore, when a certain value of total crosstalk is assumed, the contour line with a fixed crosstalk level can be plotted as the function of $\frac{\delta t_{f}}{\Delta T}$ and $\frac{\delta \omega_{f}}{\Delta \Omega}$ as shown by the solid line in Fig. 3 .

On the other hand, the spectral efficiency is also expressed in terms of $\frac{\delta \omega_{f}}{\Delta \Omega}$ and $\frac{\delta t_{f}}{\Delta T}$, because the relation $\frac{\delta \omega_{f}}{\Delta \Omega}$. $\frac{\delta t_{f}}{\Delta T}=\frac{\delta v_{f}}{\Delta v} \cdot \frac{\delta t_{f}}{\Delta T}=\frac{0.441}{\Delta v \Delta T}$ can be derived from the transform limit condition for the Gaussian filter response without dispersion and $\frac{1}{\Delta v \Delta T}$ is the spectral efficiency. This relation is expressed by hyperbolas as shown by the dashed curve in Fig. 4. Therefore, both the total crosstalk and the spectral efficiency can be plotted as the functions of $\frac{\delta t_{f}}{\Delta T}$ and $\frac{\delta \omega_{f}}{\Delta \Omega}$. Figure 3 shows the relation between the crosstalk and the spectral efficiency for which the leakage power from both adjacent channels is assumed to be the interchannel crosstalk. 


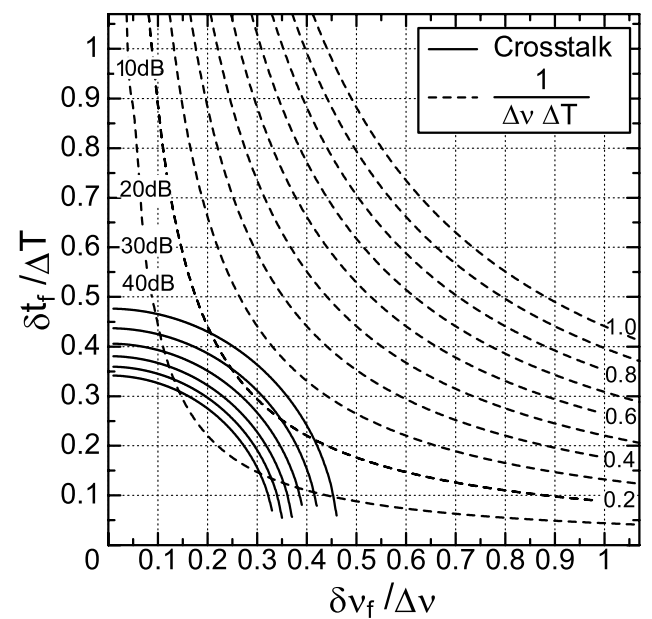

Fig. 3 Relation between spectral efficiency and crosstalk (leakage power).

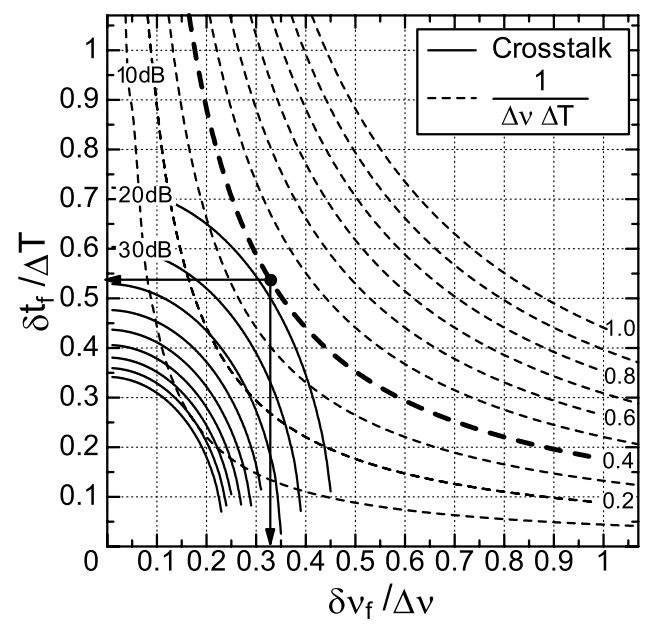

Fig. 4 Relation between spectral efficiency and crosstalk (coherent crosstalk).

\subsection{Coherent Crosstalk}

Next, we consider the case when the interchannel crosstalk is given by the worst case of coherent crosstalk. In this case, the optimum crosstalk condition is derived from Eqs. (8) and (10) as follows:

$$
\left|\exp \left[-\frac{4 \ln \sqrt{2}}{\left(\frac{\delta t_{s}}{\Delta T}\right)^{2}+\left(\frac{\delta t_{f}}{\Delta T}\right)^{2}}\right]\right|^{2}=4 \exp \left[-\frac{4 \ln \sqrt{2}}{\left(\frac{\delta \omega_{s}}{\Delta \Omega}\right)^{2}+\left(\frac{\delta \omega_{f}}{\Delta \Omega}\right)^{2}}\right] .
$$

By solving this equation and representing $\frac{\delta \omega_{s}}{\Delta \Omega}$ by $\frac{\delta t_{s}}{\Delta T}$, the optimum condition can be obtained as

$$
\left(\frac{\delta t_{s}}{\Delta T}\right)^{2}=\frac{2\left(\frac{\delta \omega_{f}}{\Delta \Omega}\right)^{2}+\left(\frac{\delta \omega_{f}}{\Delta \Omega}\right)^{2}\left(\frac{\delta t_{f}}{\Delta T}\right)^{2}}{1-\left(\frac{\delta \omega_{f}}{\Delta \Omega}\right)^{2}} .
$$

The relation between $\frac{\delta \omega_{f}}{\Delta \Omega}$ and $\frac{\delta t_{f}}{\Delta T}$ is expressed by

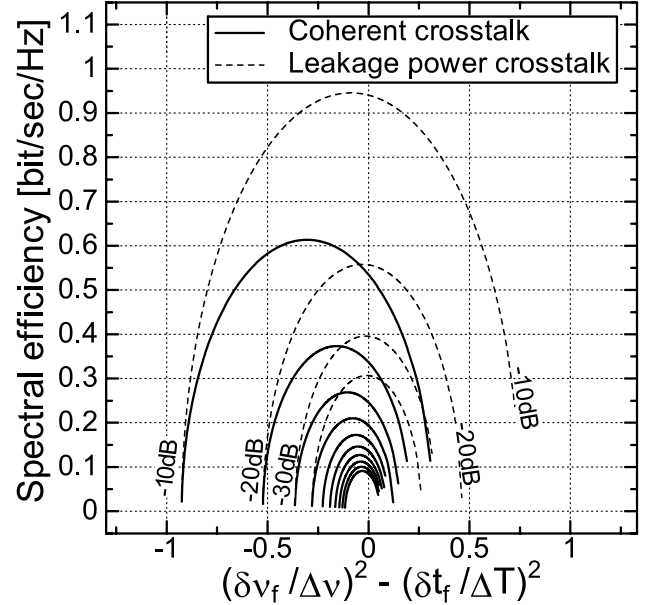

Fig.5 Conformal mapping chart showing maximum spectral efficiency and crosstalk for Gaussian filter.

$$
\begin{aligned}
\left(\frac{\delta t_{f}}{\Delta T}\right)^{2}= & \frac{40 \log 2}{10 \log 2-C T^{(d B)}} \\
& -\left(\frac{\delta \omega_{f}}{\Delta \Omega}\right)^{2}\left(2+\frac{40 \log 2}{10 \log 2-C T^{(d B)}}\right) .
\end{aligned}
$$

The crosstalk given by Eq. (15) and the spectral efficiency are plotted in Fig. 4. Due to the coherent crosstalk, the crosstalk level giving a fixed spectral efficiency is smaller than that shown in Fig. 3.

In addition, Fig. 5 is the conformal transformation of Figs. 3 and 4 by $W=A z^{2}(z=x+j y, x$ and $y$ are the horizontal and vertical axes, respectively). We can easily obtain the optimum condition of $\frac{\delta v_{f}}{\Delta v}$ and $\frac{\delta t_{f}}{\Delta T}$ to maximize the spectral efficiency for a given crosstalk level. In Fig. 5, the dashed line represents the calculated results for the case that the leakage power is the dominant interchannel crosstalk, and the solid line represents those for the case that the coherent crosstalk is the dominant interchannel crosstalk. It can be seen from Fig. 5 that the coherent crosstalk dominates the interchannel crosstalk compared with the leakage power from adjacent ports.

Using this analysis, the filter response shape can be optimally designed to achieve the ultimate spectral efficiency under a given condition of crosstalk, and this will be presented in Sects. 4-6.

\subsection{Optimum Design of Filter Bandwidth and Pulse Width}

Let us consider the actual WDM system design, that is, the optimum FWHM of the filter spectrum and the input pulse width. For example, in the $40 \mathrm{Gbit} / \mathrm{sec}$ WDM system with $100 \mathrm{GHz}$ spacing, the spectral efficiency $=0.4$. When the worst case of coherent crosstalk is assumed as the interchannel crosstalk, the total crosstalk level is determined to be $18 \mathrm{~dB}$ from Fig. 5. The values of $\frac{\delta v_{f}}{\Delta v}$ and $\frac{\delta t_{f}}{\Delta T}$ to maximize the spectral efficiency are determined to be 0.33 and 0.54 , respectively from Fig. 4. Substituting these values into Eq. (14), we obtain the value of $\frac{\delta t_{s}}{\Delta T}$ to be 0.35 . Thus, the 
FWHM of the filter spectrum and the pulse width to maximize the spectral efficiency are determined to be $33 \mathrm{GHz}$ and 8.8 psec, respectively.

\section{Relation between Crosstalk and Spectral Efficiency for Box-Like Filter}

The spectrum response of a filter is generally required to be close to the box-like shape, and it is important to compare the ultimate spectral efficiency for the box-like spectrum to that for the Gaussian filter. Therefore, we analyzed the relation between the spectral efficiency and the crosstalk by changing the filter spectrum shape.

The considered spectrum response is expressed by

$$
H(\omega)=A \exp \left[b\left(\omega-\omega_{0}\right)^{\alpha}\right],
$$

where $\alpha=2,4,10, \infty$. The case of $\alpha=2$ is the Gaussian filter, and increasing the value of $\alpha$, the pass band is flattened and the roll-off is sharpened, that is, the spectrum approaches the rectangular shape. $\alpha=\infty$ corresponds to the absolutely rectangular filter spectrum. Figure 6 shows the spectrum responses for different values of $\alpha$, where the FWHMs of the spectrum are all the same as $50 \mathrm{GHz}$ $(0.4 \mathrm{~nm})$. The impulse responses are also shown and compared in Fig. 7. It is seen from Fig. 7 that, when $\alpha$ is larger than 2, the side lobes arise in the impulse response, and this side lobe level increases by increasing the value of $\alpha$.

Figures 8 and 9 show the comparison of the spectral efficiency for different values of $\alpha$, when the total crosstalk (the sum of intersymbol interference and interchannel crosstalk) is assumed to be $-10 \mathrm{~dB}$ and $-20 \mathrm{~dB}$, respectively. The coherent crosstalk between adjacent channels is considered as the interchannel crosstalk. Although a high spectral efficiency is obtained by box-like filter spectrum, discontinuities arise in the curves of the spectral efficiency in Fig. 9. This is because the side-lobes arise in the output pulse of the filter, and when the input pulse width is narrow (the input spectrum is wider than the filter response), the side-lobe level increases and exceeds $-23 \mathrm{~dB}$

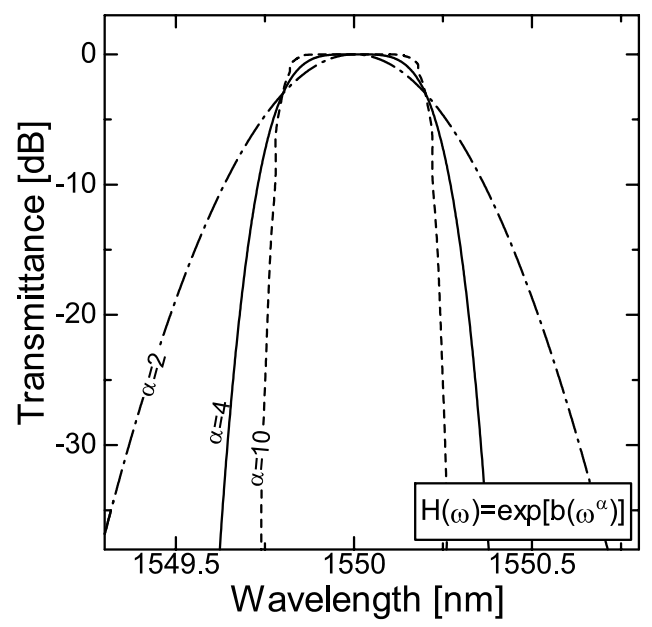

Fig. 6 Spectrum responses for exponential filters

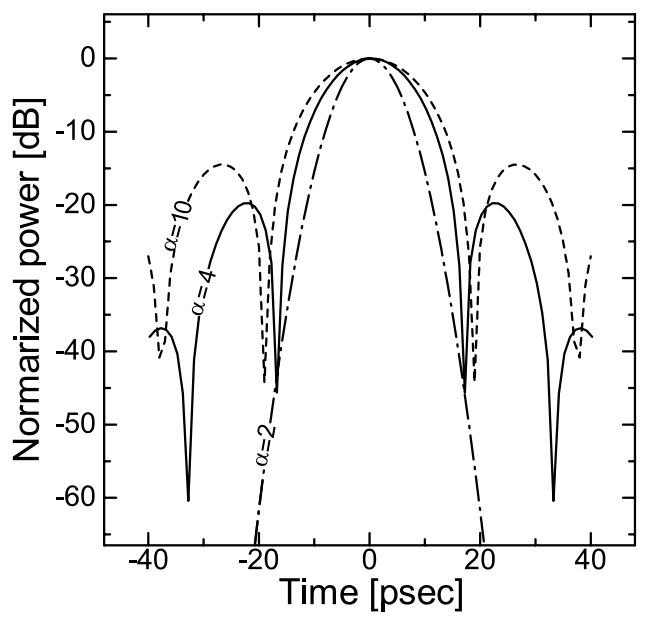

Fig. 7 Impulse responses for exponential filters.

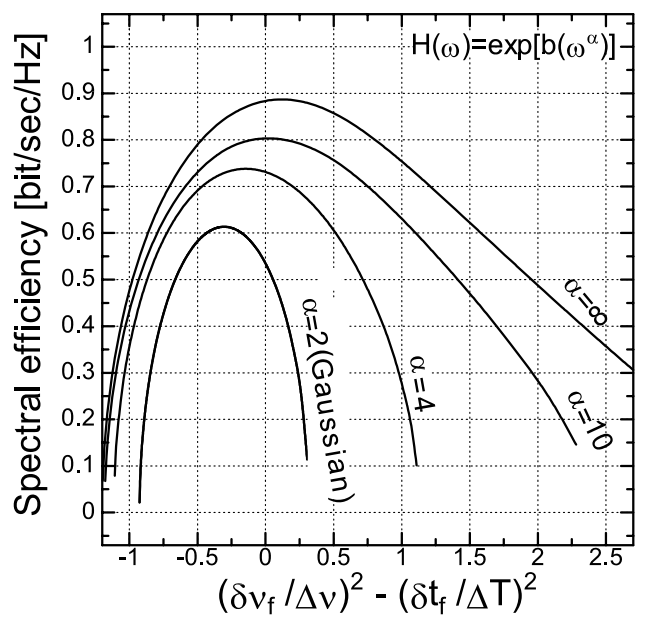

Fig. 8 Comparison of spectral efficiency for different values of $\alpha$ (crosstalk level is $-10 \mathrm{~dB}$ ).

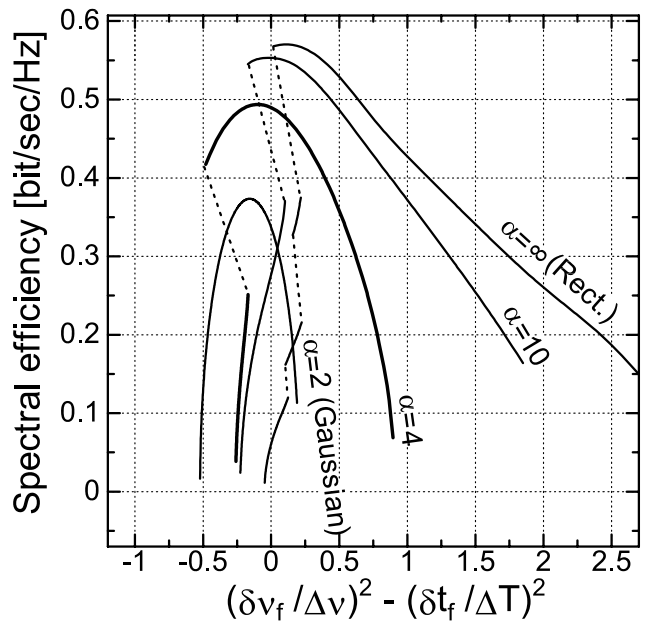

Fig. 9 Comparison of spectral efficiency for different values of $\alpha$ (crosstalk level is $-20 \mathrm{~dB}$ ). 


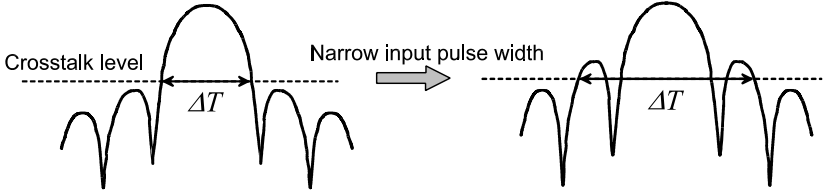

Fig. 10 Side lobe in output pulse.

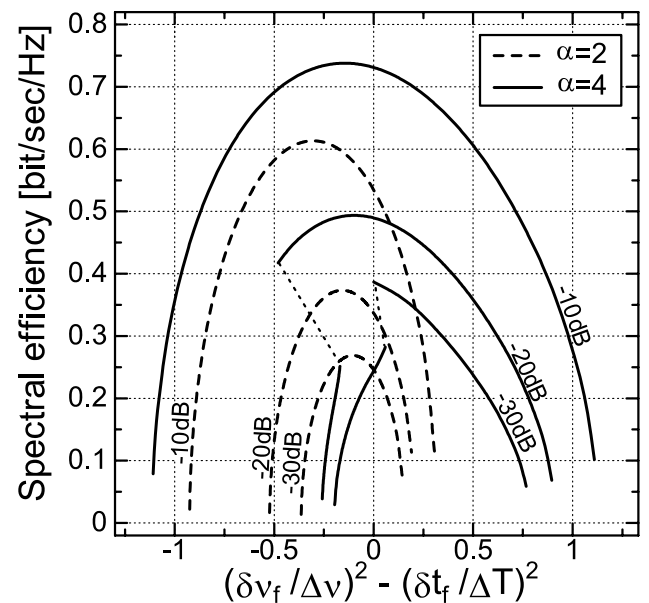

Fig. 11 Relation between spectral efficiency and crosstalk for $\alpha=2$ and

$\left(C T^{(T)}=C T^{(v)}=-23 \mathrm{~dB}\right.$ and total crosstalk level is $-20 \mathrm{~dB}$ ). Due to this side-lobe, the optimum pulse interval $\Delta T$ leaps when the input pulse width becomes narrower than a certain value, as shown in Fig. 10. In this case, the spectral efficiency rapidly deteriorates. The spectral efficiency increases when the value of $\alpha$ increases, i.e. the filter spectrum shape becomes box-like. However, the curve of spectral efficiency becomes discontinuous at some points due to the side-lobe of the output pulse. Figure 11 shows the relation between the spectral efficiency and the crosstalk for filter responses of $\alpha=4$. For comparison, the relation for the Gaussian filter $(\alpha=2)$ is also plotted by the broken line in Fig. 11. The ultimate spectral efficiency for the filter of $\alpha=4$ is higher than that of Gaussian filter when the crosstalk level is $-10 \mathrm{~dB}$. However, when the crosstalk level is lower than $-20 \mathrm{~dB}$, discontinuities arise in the curve of the spectral efficiency for $\alpha=4$.

\section{Improvement of Spectral Efficiency by Filter Cas- cading}

When the filter spectrum is close to rectangular, the curve of the spectral efficiency involves some discontinuous points, and this discontinuity deteriorates the spectral efficiency. To avoid the discontinuity, we propose a cascaded filter of $\alpha=2$ and $\alpha=4$. As a characteristic of the filter, the Gaussian filter has a small roll-off. Thus, the interchannel crosstalk is large, but the output pulse has no side-lobe. On the other hand, the exponential filter with $\alpha=4$ has a large roll-off, but the side-lobe arises in the output pulse, which requires the large pulse interval. The cascaded filter has a Gaussian response

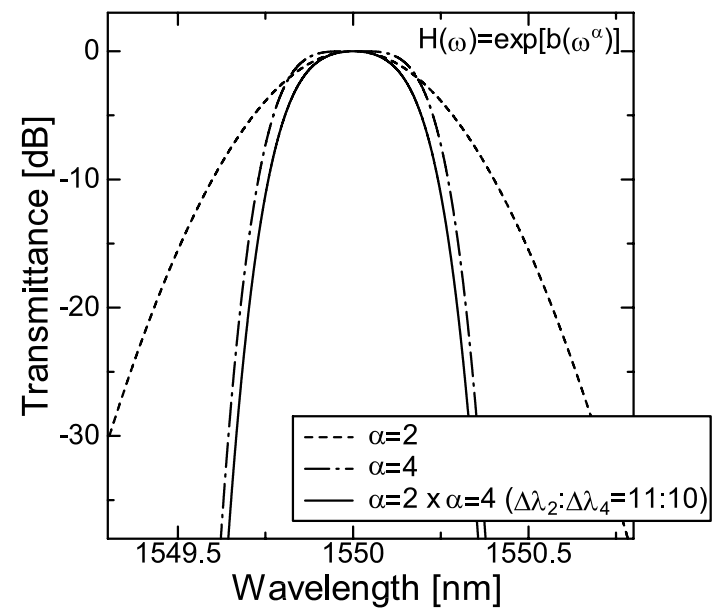

Fig. 12 Spectrum responses of cascaded and exponential filters.

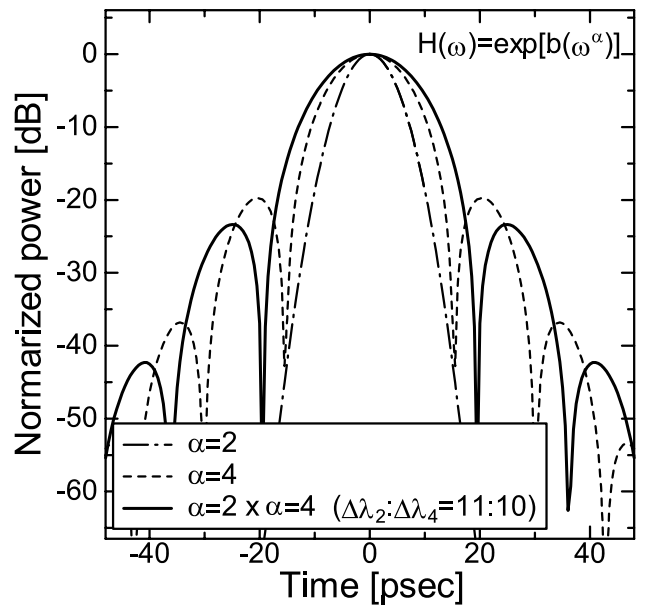

Fig. 13 Impulse responses of cascaded and exponential filters.

near the center wavelength, so that the side lobe in the output pulse can be suppressed, and has large roll-off out of band, so that the interchannel crosstalk can be decreased.

The spectrum response and the impulse response of the cascaded filter are shown in Figs. 12 and 13, respectively, where the ratio of FWHMs is $\delta v_{\alpha=2}: \delta v_{\alpha=4}=11: 10$. The ratio of the FHWMs of $\delta v_{\alpha=2}$ and $\delta v_{\alpha=4}$ was optimized considering on the side-lobe level of the impulse response of the cascaded filter. Figure 14 shows the comparison of the side-lobe level for different FWHM ratios of $\delta v_{\alpha=2}$ and $\delta v_{\alpha=4}$. To obtain the continuous spectral efficiency for the total crosstalk of $-20 \mathrm{~dB}$, the allowable side-lobe level is $-23 \mathrm{~dB}$ (the sum of intersymbol interference and interchannel crosstalk is $-20 \mathrm{~dB}$ ), while the case of much lower sidelobe level corresponds to the wider impulse response width. Thus, the output pulse width is also wider and results in low spectral efficiency. From Fig. 14, it can be seen that the optimum ratio is $\delta v_{\alpha=2}: \delta v_{\alpha=4}=11: 10$ for the side-lobe level of less than $-23 \mathrm{~dB}$. From Fig. 13, the side lobe level is larger than $-20 \mathrm{~dB}$ for $\alpha=4$, while for the cascaded filter, the sidelobe level is decreased to less than $-23 \mathrm{~dB}$. 


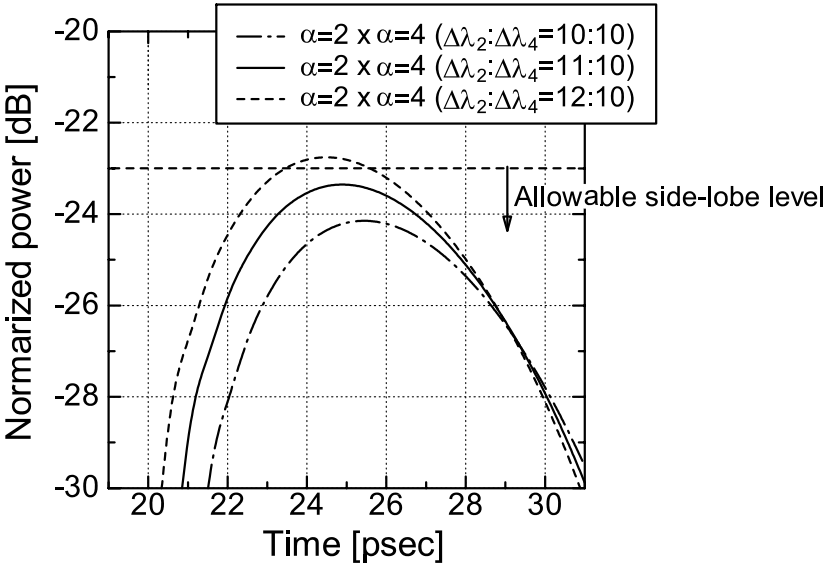

Fig. 14 Comparison of side-lobe level of cascaded exponential filters.

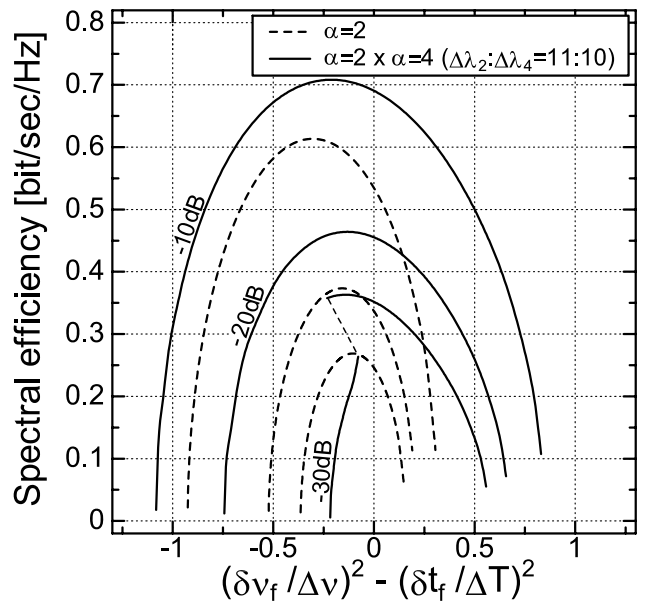

Fig. 15 Relation between spectral efficiency and crosstalk of cascaded exponential filter.

Figure 15 shows the relation between the spectral efficiency and crosstalk of the cascaded filter response. It can be seen from this figure that the cascaded filter can achieve a higher spectral efficiency than that of the spectrum response of a conventional Gaussian filter, and the discontinuity in the curve of spectral efficiency can be inhibited for the crosstalk level of $-20 \mathrm{~dB}$. The curve of spectral efficiency without discontinuity means that a large tolerance for filter spectrum response is needed to obtain high spectral efficiency.

Similar to the Gaussian filter, we can design the optimum allocation of the filter spectrum width and the input pulse width. For example, the $40 \mathrm{Gbit} / \mathrm{sec}$ system with $100 \mathrm{GHz}$ spacing, which corresponds to the spectral efficiency of 0.4 , was considered. In this case, from Fig. 15, the total crosstalk level was reduced to $-26 \mathrm{~dB}$ from $-18 \mathrm{~dB}$ of the Gaussian filter. Figures 16 and 17 show the relation between the crosstalk and spectral efficiency for filter responses and for the signal parameters, respectively. The optimum frequency occupation $\frac{\delta v_{f}}{\Delta v}$ is determined to be 0.44 from Fig. 16, and the optimum time duty ratio $\left(\frac{\delta t_{s}}{\Delta T}\right)$ is determined to be 0.53 from Fig. 17, to minimize the crosstalk.

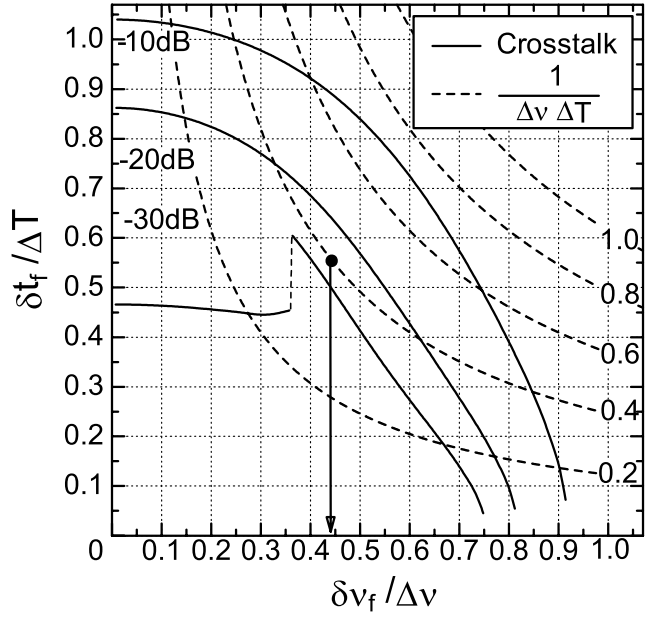

Fig. 16 Determination of $\delta v_{f}$ from the relation between crosstalk and spectral efficiency.

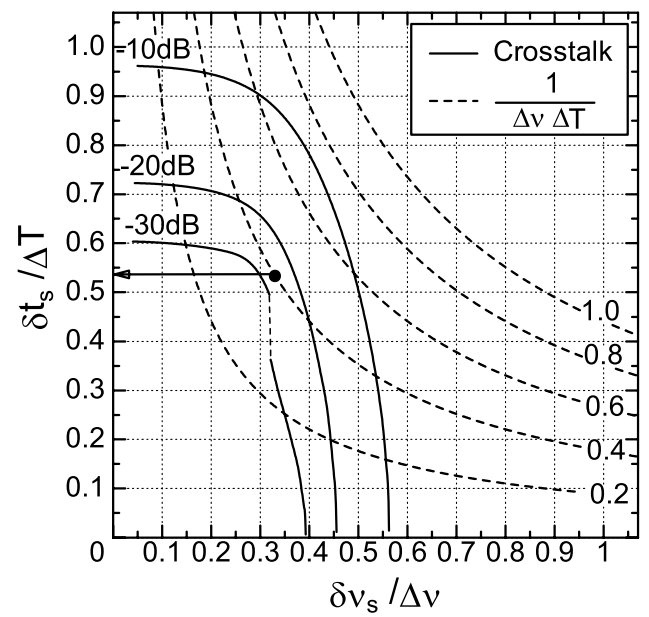

Fig. 17 Determination of $\delta t_{s}$ from the relation between crosstalk and spectral efficiency.

Thus, we can design the optimum spectrum width of the filter and the input pulse width to be $44 \mathrm{GHz}$ and $13 \mathrm{psec}$ $(25 \mathrm{psec} \times 0.53)$, respectively.

\section{Impact of Rejection Floor}

The rejection floor inheres in the actual wavelength filter satisfying the causality, but there has been no consideration of the floor level which might deteriorate the spectral efficiency. Thus, we clarified the ultimate spectral efficiency limited by the rejection floor of Gaussian filter response (AWG).

First, we considered the impact of the rejection floor in adjacent channels on the spectral efficiency. As seen from Fig. 5, the coherent crosstalk dominates the interchannel crosstalk compared with the leakage power from adjacent channels. In the previous analysis, the rejection of the filter was assumed to reach zero $(-\infty[\mathrm{dB}])$ with the increase of the detuning of the frequency. However, the actual filter 


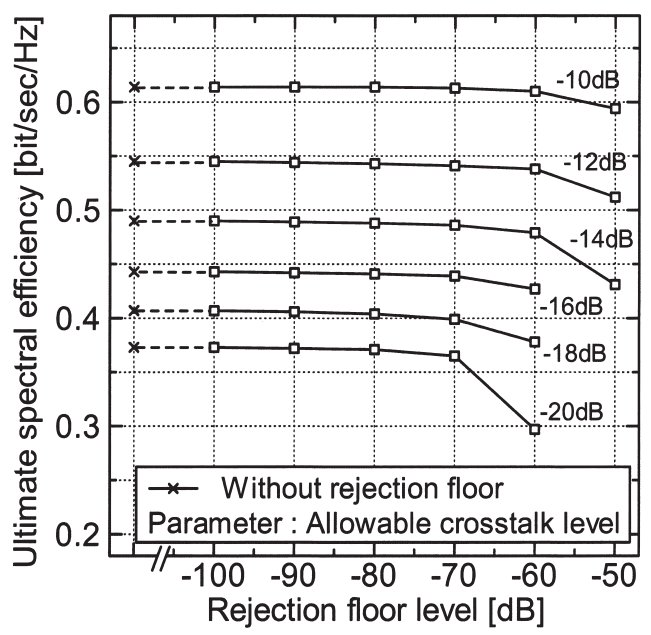

Fig. 18 Deterioration of spectral efficiency by rejection floor of the nearest adjacent channels (coherent crosstalk is dominant).

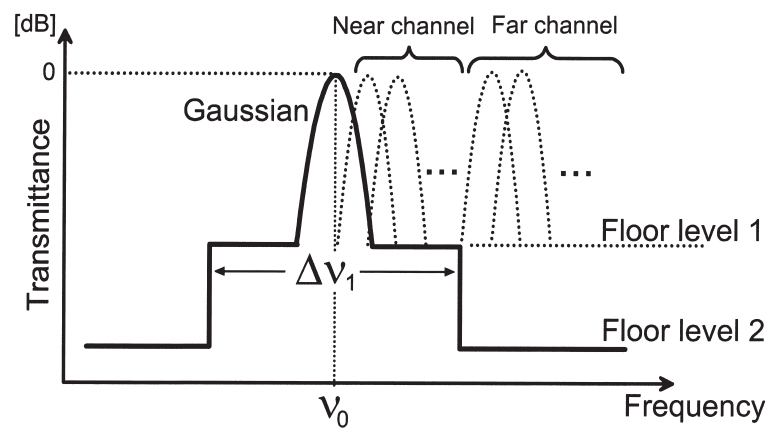

Fig. 19 Proposed filter spectrum response with rejection floor.

must have so-called rejection floor in the rejection band to satisfy the causality, and the crosstalk in the DWDM system may accumulate with increasing the number of channels. The rejection floor is sometimes called crosstalk floor, but this terminology is not correct. The crosstalk is the product of the filter spectrum response of an interested channel and the signal spectrum of the other channels in the rejection band of the filter. Figure 18 shows the deterioration of the spectral efficiency by the increase of floor level in the filter. In this analysis, the coherent crosstalk was considered as the interchannel crosstalk. It can be seen that when the floor level increases beyond $-60 \mathrm{~dB}$, the efficiency rapidly decreases. Therefore, the floor level must be lower than $-70 \mathrm{~dB}$. The cascading of filter can realize this low floor level when it is difficult to reduce the floor level to $-70 \mathrm{~dB}$ by a single filter.

Next, we evaluated the impact of the multi-crosstalk channels, excluding adjacent channels. Figure 19 shows the filter spectrum proposed in this analysis. It has the Gaussian function around the center wavelength and the two rejection floor levels in the rejection band. The floor level 1 has a higher crosstalk level near by the center wavelength, while the floor level 2 has lower level far from that. This spectrum response can be realized by cascading a Gaussian filter with

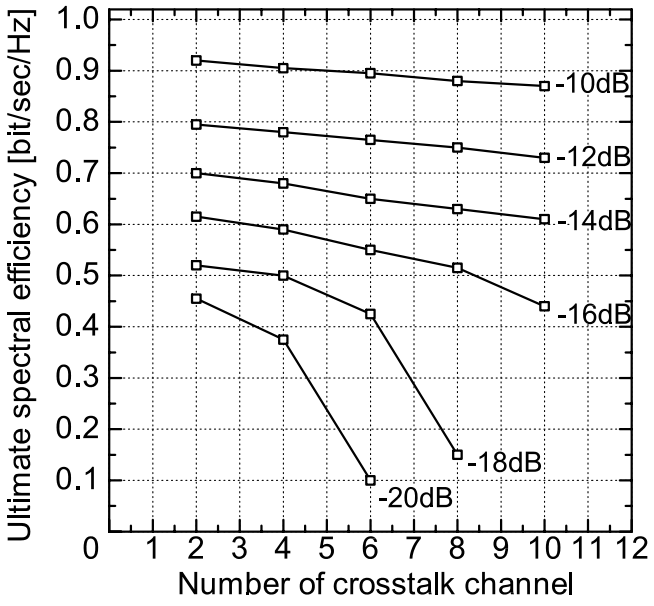

Fig. 20 Deterioration of spectral efficiency vs. number of leakage channels (Floor level $1=-30 \mathrm{~dB}$ ).

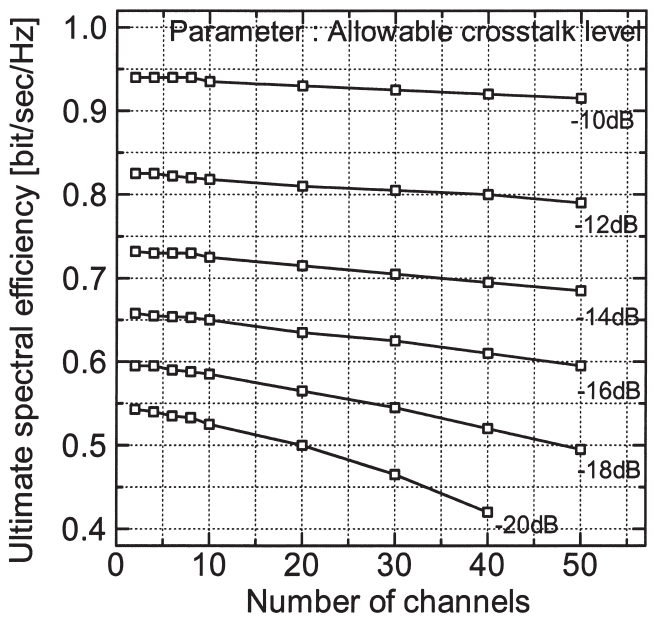

Fig. 21 Deterioration of spectral efficiency vs. number of leakage channels (Floor level $1=-40 \mathrm{~dB}$ ).

rejection floor and a box-like filter with wider bandwidth. When we analyze the influence of the crosstalk channels excluding the nearest adjacent channels, we need to consider only the accumulation of leakage power from other channels. This is because the crosstalk channels are far from the center wavelength by more than $100 \mathrm{GHz}$, and therefore the photo-detector can not respond to the intermediate frequency of the coherent crosstalk.

First, let us consider the floor level 1. Figures 20 and 21 show the deterioration of the spectral efficiency vs. the increase of the number of channels transmitted in the floor level 1. The floor level 1 was assumed to be $-30 \mathrm{~dB}$ for Fig. 20 and $-40 \mathrm{~dB}$ for Fig. 21, respectively. It can be seen from Fig. 20, the deterioration of the spectral efficiency is large with the increase of the number of crosstalk channels when the floor level 1 is $-30 \mathrm{~dB}$. From Fig. 21, the allowable number of channels must be less than 8 channels to keep the deterioration of the spectral efficiency less than 0.01 . Thus, the required floor level 1 is clarified to be less than $-40 \mathrm{~dB}$.

Next, we evaluate the requirement of the floor level 2. 


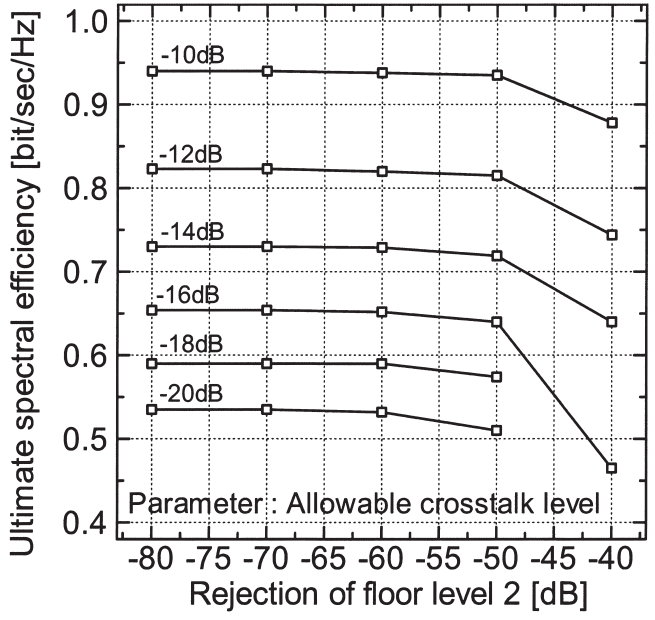

Fig. 22 Spectral efficiency vs. the rejection of floor level 2(Floor level 1 $=-40 \mathrm{~dB} \times 6$ channels $)$.

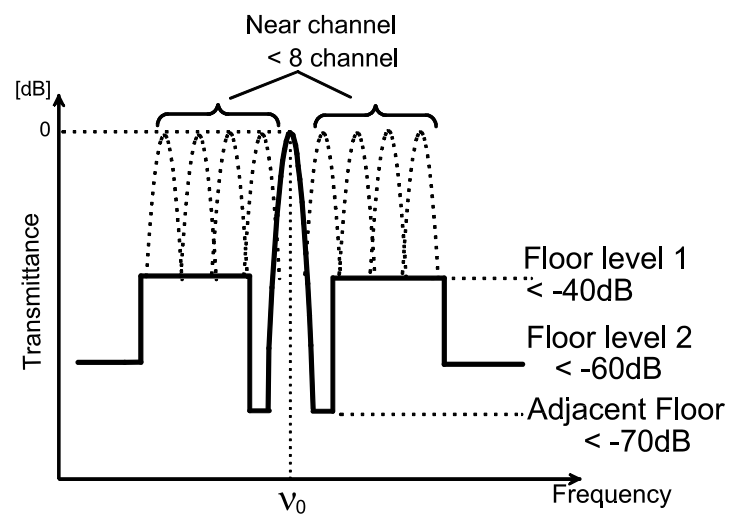

Fig. 23 Required rejection floor label of wavelength filter.

In the calculation, we assume the number of crosstalk channels transmitted in the band of floor level $1(-40 \mathrm{~dB})$ to be 6 channels $\left(\delta v_{1}=6 \Delta v_{1}\right)$ and the number of channels transmitted in the band of the floor level 2 to be 100 channels. Figure 22 shows the change of spectral efficiency by changing the level of rejection floor 2. From Fig. 22, it is seen that when the floor level is greater than $-50 \mathrm{~dB}$, the efficiency rapidly deteriorates. Thus, we must reduce the floor level 2 to be less than $-60 \mathrm{~dB}$.

Consequently, the required rejection floor of the filter spectrum response is summarized in Fig. 23, in which the adjacent floor level must be less than $-70 \mathrm{~dB}$, the allowable number of near channels is less than 8 for $-40 \mathrm{~dB}$ of floor level 1 , and the floor level 2 must be less than $-60 \mathrm{~dB}$.

\section{Conclusion}

A method to analyze the relation between the spectral efficiency and the crosstalk in DWDM systems was proposed. Two kinds of crosstalks, the intersymbol interference and the interchannel crosstalk, were considered, and the optimum crosstalk condition was defined so that both crosstalk levels are equal. Assuming a Gaussian function as the filter spectrum response and the pulse shape, the optimum condition was analytically obtained. Under this optimum condition, the relation between the spectral efficiency and the total crosstalk level was clarified by expressing them as the functions of $\frac{\delta v_{f}}{\Delta v}$ and $\frac{\delta t_{f}}{\Delta T}$. In addition, it was clarified that the coherent crosstalk dominates the interchannel crosstalk compared with the leakage power from adjacent channels.

This analytical method was applied to the box-like filter response. It was clarified that the spectral efficiency is higher than that of Gaussian filter response, however, a discontinuity arises and the efficiency deteriorates rapidly at the leap point. To avoid this discontinuity, the cascading of the two different spectrum responses was proposed, and the discontinuity of the curve of spectral efficiency can be eliminated while maintaining the high spectral efficiency.

The impact of the rejection floor in the filter spectrum response on the spectral efficiency was also analyzed, and the required rejection floor was clarified.

In the actual transmission system, the ultimate spectral efficiency should be defined as the maximum spectral efficiency for a given bit error rate. Therefore, we are now analyzing the relationship between the ultimate spectral efficiency, and the eye-opening penalty and the result will be presented in future.

\section{References}

[1] K. Fukuchi, T. Kasamatsu, M. Morie, T. Ito, K. Sekiya, D. Ogasahara and T. Ono, "10.92-Tb/s $(273 \times 40-\mathrm{Gb} / \mathrm{s})$ triple-band/ultra-dense WDM optical-repeatered transmission experiment," OFC2001, PD24, March 2001.

[2] S. Bigo, Y. Frignac, G. Charlet, W. Idler, S. Borne, H. Gross, R. Discheler, W. Poehlmann, P. Tran, C. Simonneau, D. Bayart, G. Veith, A. Jouudan, and J. Hamaide, "10.2 Tbit/s (256×42.7 Gbit/s PDM/WDM) transmission over $100 \mathrm{~km}^{\text {TeraLight }}{ }^{T M}$ fiber with $1.28 \mathrm{bit} / \mathrm{s} / \mathrm{Hz}$ spectral efficiency," OFC2001, PD25, March 2001.

[3] H. Sotobayashi, W. Chujo, and K. Kitayama, "1.6 bit/s/Hz, $6.4 \mathrm{Tbit} / \mathrm{s}$ $\mathrm{OCDM} / \mathrm{WDM}(4 \mathrm{OCDM} \times 40 \mathrm{WDM} \times 40 \mathrm{Gbit} / \mathrm{s})$ transmission $\mathrm{ex}-$ periment," ECOC'01, PD.M.1.3, Oct. 2001.

[4] K.-P. Ho and J.M. Kahn, "Ultimate spectral efficiency limits in DWDM systems," OFC2002, 12B22-1, July 2002.

[5] C.E. Shannon, "A mathematical theory of communication," Bell Syst. Tech. J., vol.27, no.3, pp.379-423, pp.623-656, July 1948.

[6] H. Nyquist, "Certain topics in telegraph transmission theory," Trans. A.I. E.E., vol.47, pp.617-644, Feb. 1928.

[7] S. Suzuki and Y. Kokubun, "Design rule of wavelength filter bandwidth and pulse width for ultimate spectral efficiency limited by crosstalk in DWDM systems," Photon. Tech. Lett., vol.15, no.11, pp.1645-1647, 2003.

\section{Appendix: Derivation of Eqs. (8), (9), and (10)}

Since the pulse interval is $\Delta T$, the intersymbol interference is calculated by the ratio $\frac{\left|f_{\text {out }}^{(s)}(\Delta T)\right|^{2}}{\left|f_{\text {out }}^{(s)}(0)\right|^{2}}$ and is expressed by

$$
C T^{(T)}=\left|\exp \left[-\frac{1}{\frac{1}{a}+\frac{1}{b}} \Delta T^{2}\right]\right|^{2}
$$




$$
=\left|\exp \left[-\frac{4 \ln \sqrt{2}}{\left(\frac{\delta t_{s}}{\Delta T}\right)^{2}+\left(\frac{\delta t_{f}}{\Delta T}\right)^{2}}\right]\right|^{2} .
$$

Next, the leakage spectrum from $\omega_{0}+\Delta \Omega$ can be obtained by the product of the filter spectrum response and the signal spectrum of which center angular frequency is different by the channel spacing $\Delta \Omega$. Calculating the inverse Fourier transform of this spectrum, we obtain the output crosstalk pulse as

$$
\begin{aligned}
f_{\text {out }}^{(+\Delta \Omega)}(t)= & \frac{1}{\sqrt{1+\frac{a}{b}}} \exp \left[-\frac{1}{\frac{1}{a}+\frac{1}{b}} t^{2}\right] \exp \left[-\frac{\Delta \Omega^{2}}{4 a+4 b}\right] \\
& \left.\cdot \exp \left[j\left(\omega_{0}-\frac{b}{a+b} \Delta \Omega\right) t+\phi\right], \quad \text { (A- } 2\right)
\end{aligned}
$$

where $\phi$ is the phase difference of the carrier between signal channel and $+\Delta \Omega$ channel. In a similar way, the leakage signal from $\omega_{0}-\Delta \Omega$ can be obtained by substituting $-\Delta \Omega$ instead of $+\Delta \Omega$. Thus, the crosstalk by the leakage power from one adjacent channel $C T^{(L P)}$ is obtained by $\frac{\left|f_{\text {out }}^{(+\Delta)}(t)\right|^{2}}{\mid f_{\text {out }}^{(s)}(t)^{2}}$,

$$
\begin{aligned}
C T^{(L P)} & =\left|\exp \left[-\frac{\Delta \Omega^{2}}{4 a+4 b}\right]\right|^{2} \\
& =\left|\exp \left[-\frac{4 \ln \sqrt{2}}{\left(\frac{\delta \omega_{s}}{\Delta \Omega}\right)^{2}+\left(\frac{\delta \omega_{f}}{\Delta \Omega}\right)^{2}}\right]\right|^{2} .
\end{aligned}
$$

Now, when the states of the original channel and $\pm \Delta \Omega$ channels are "1" and the power levels of individual frequency channels are also the same, the received intensity is given by

$$
\begin{aligned}
\left|f_{\text {out }}(t)\right|^{2}= & \left|f_{\text {out }}^{(s)}(t)+f_{\text {out }}^{(+\Delta \Omega)}(t)+f_{\text {out }}^{(-\Delta \Omega)}(t)\right|^{2} \\
\simeq & \left|f_{\text {out }}^{(s)}(t)\right|^{2}+\left|f_{\text {out }}^{(+\Delta \Omega)}(t)\right|^{2}+\left|f_{\text {out }}^{(-\Delta \Omega)}(t)\right|^{2} \\
& \frac{+f_{\text {out }}^{(s)}(t) \cdot\left(f_{\text {out }}^{(+\Delta \Omega)}(t)^{*}+f_{\text {out }}^{(-\Delta \Omega)}(t)^{*}\right)}{} \\
& \frac{+f_{\text {out }}^{(s)}(t)^{*} \cdot\left(f_{\text {out }}^{(+\Delta \Omega)}(t)+f_{\text {out }}^{(-\Delta \Omega)}(t)\right)}{}
\end{aligned}
$$

The underlined terms of Eq. (A.6) causes the coherent crosstalk and is rewritten as follows:

$$
\begin{array}{r}
\begin{aligned}
f_{\text {out }}^{(s)}(t) & \cdot f_{\text {out }}^{(+\Delta \Omega)}(t)^{*}+f_{\text {out }}^{(s)}(t)^{*} \cdot f_{\text {out }}^{(+\Delta \Omega)}(t) \\
& =2 A B \cos \left[\left(\frac{b}{a+b} \Delta \Omega\right) t+\phi_{+}\right], \\
f_{\text {out }}^{(s)}(t) & \cdot f_{\text {out }}^{(-\Delta \Omega)}(t)^{*}+f_{\text {out }}^{(s)}(t)^{*} \cdot f_{\text {out }}^{(-\Delta \Omega)}(t) \\
= & 2 A B \cos \left[\left(\frac{b}{a+b} \Delta \Omega\right) t+\phi_{-}\right],
\end{aligned}
\end{array}
$$

$$
B=\frac{1}{\sqrt{1+\frac{a}{b}}} \exp \left[-\frac{1}{\frac{1}{a}+\frac{1}{b}} t^{2}\right] \exp \left[-\frac{\Delta \Omega^{2}}{4 a+4 b}\right] .
$$

Here it should be noted that the electric angular frequency of the coherent crosstalk is not $\Delta \Omega$ but $\frac{b}{a+b} \Delta \Omega$. This means that the photo detector can respond to this frequency in the WDM system with high spectral efficiency, and the coherent crosstalk may affect the main signal.

Coherent crosstalk $C T^{(C C)}$ is given as the ratio of Eq. (A. 7) or (A. 8) to $\left|f_{\text {out }}^{(s)}(t)\right|^{2}$ at $t=0$ (timing of symbol determination). Thus by dividing Eq. (A. 7) and Eq. (A. 8) by $\left|f_{\text {out }}^{(s)}(t)\right|^{2}=A^{2}$ and substituting $t=0$, we obtain the coherent crosstalk affected by adjacent channels as follows:

$$
\begin{aligned}
C T^{(C C+)} & =\frac{2 A B}{A^{2}} \cos \phi_{+} \\
& =2 \exp \left[-\frac{\Delta \Omega^{2}}{4 a+4 b}\right] \cos \phi_{+} \\
& =2 \exp \left[-\frac{4 \ln \sqrt{2}}{\left(\frac{\delta \omega_{s}}{\Delta \Omega}\right)^{2}+\left(\frac{\delta \omega_{f}}{\Delta \Omega}\right)^{2}}\right] \cos \phi_{+} \\
C T^{(C C-)} & =2 \exp \left[-\frac{4 \ln \sqrt{2}}{\left(\frac{\delta \omega_{s}}{\Delta \Omega}\right)^{2}+\left(\frac{\delta \omega_{f}}{\Delta \Omega}\right)^{2}}\right] \cos \phi_{-} .
\end{aligned}
$$

Here, the intersymbol interference and the leakage power from adjacent channels cause the bit error from " 0 " to " 1 ," while the coherent crosstalk causes the bit error from " 1 " to " 0 ," because it occurs when the signal channel and its adjacent channels are both "1." This may take the negative value depending on $\phi_{+}$and $\phi_{-}$as seen from Eqs. (A.9) and (A-10). The coherent crosstalk has the biggest influence on bit error when $\phi_{+}= \pm \pi$ or $\phi_{-}= \pm \pi$, and the worst value of coherent crosstalk is

$$
C T^{(C C)}=4 \exp \left[-\frac{4 \ln \sqrt{2}}{\left(\frac{\delta \omega_{s}}{\Delta \Omega}\right)^{2}+\left(\frac{\delta \omega_{f}}{\Delta \Omega}\right)^{2}}\right] .
$$

where

$$
A=\frac{1}{\sqrt{1+\frac{a}{b}}} \exp \left[-\frac{1}{\frac{1}{a}+\frac{1}{b}} t^{2}\right]
$$

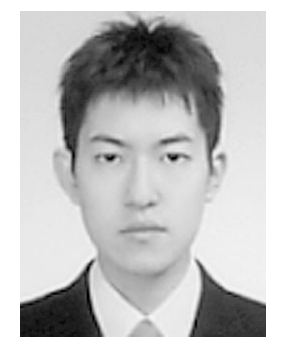

Shuichi Suzuki was born in Kanagawa, Japan, on October 6, 1976. He received the B.E. and M.E. degrees in electrical and computer engineering from Yokohama National University, Yokohama, Japan, in 1999 and 2001, respectively. He is currently working toward the Ph.D. degree at Yokohama National University. His research interests include waveguide-type functional devices. 


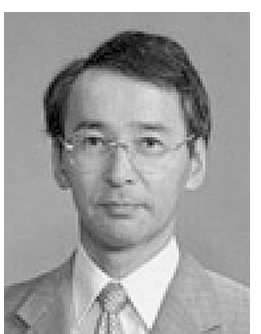

Yasuo Kokubun was born in Fukushima, Japan, on July 7, 1952. He received the B.E. degree from Yokohama National University, Yokohama, Japan, in 1975 and the M.E. and Dr.Eng. degrees from Tokyo Institute of Technology, Tokyo, Japan, in 1977 and 1980, respectively. From 1980 to 1983 , he was with the Research Laboratory of Precision Machinery and Electronics, Tokyo Institute of Technology, as a Research Associate. In 1983, he joined Yokohama National University as an Associate. In 1983, he joined Yokohama National University as an Associate Professor and is now a Professor of the Department of Electrical and Computer Engineering. His current research is in integrated photonics, especially waveguide-type. Up to 1985, he was with AT\& T Bell Laboratories, Holmdel, NJ, as a Visiting Researcher and was engaged in a novel waveguide on semiconductor substrate (ARROW) for integrated optics. From 1996 to 1999, he served as the Project Leader of the three-dimensional micro photonics project at the Kanagawa Academy of Science and Technology, Kanagawa, Japan. Prof. Kokubun is a member of the Optical Society of America (OSA) and the Japan Society of Applied Physics (JSAP). 